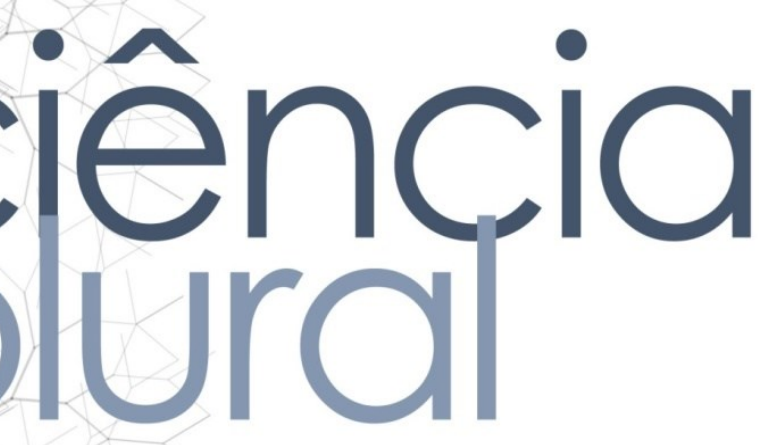

\title{
AVALIAÇÃO DE ATITUDES DIANTE DA PREVENÇÃO DE ENTEROPARASITOSES EM ESCOLARES
}

\section{Evaluation of attitudes before the prevetion enteroparasitosis in school}

Dayane Caroliny Pereira Justino - Enfermeira. Mestre em Saúde Coletiva. E-mail: daycaroliny@hotmail.com

Tainara Lorena dos Santos Ferreira - Enfermeira. Mestranda do Programa em Pós-Graduação em Saúde Coletiva na UFRN. E-mail: tainara_lorena@hotmail.com

Daísy Vieira de Araújo - Enfermeira. Mestre em Enfermagem. Professora da FACISA/UFRN. E-mail: mestredaisy@yahoo.com.br

Fábia Barbosa de Andrade - Enfermeira. Doutora em Ciências da Saúde. Professora Adjunto IV da UFRN. E-mail: fabiabarbosabr@gmail.com

Autora responsável pela correspondência:

Tainara Lorena dos Santos Ferreira. E-mail: tainara_lorena@hotmail.com. 


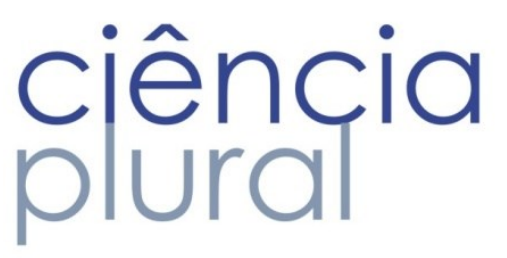

\section{RESUMO}

Introdução: As enteroparasitoses são doenças com um potencial de infecção cosmopolita, que afetam com alta frequência as faixas etárias que possuem imunidade debilitada, principalmente se os indivíduos estiverem em um ambiente propício a infecções. Todavia, esse tipo de doença pode ser evitado através de hábitos de higiene. Objetivo: Verificar o conhecimento sobre enteroparasitas e a atitude das crianças do Projeto Cidadão do Amanhã diante da prevenção de doenças. Metodologia: Trata-se de um estudo quase experimental, realizado com as crianças de 6 a 12 anos, cadastradas no Projeto Cidadão do Amanhã e atendidas na Clínica de Enfermagem da Universidade Federal do Rio Grande do Norte, ambas na cidade de Santa Cruz-RN. Utilizouse como amostra 40 crianças, sendo utilizado para coleta de dados dois instrumentos. Resultados: Verificouse o conhecimento de escolares em quatro categorias: 1) Hábitos de higiene; 2) Contaminação alimentar; 3) Autocontaminação e 4) Dermatites tópicas, observando no resultado do pós-teste aplicado ao grupo 2, melhora de acertos nas respostas, de modo que acredita-se que a educação em saúde seja a principal forma de prevenção tanto das enteroparasitoses, quanto de outras doenças que possam vir a afetar a vida não apenas do grupo aqui estudado, mas da população em geral. Conclusão: Conclui-se que há a necessidade de implantação e implementações de ações e serviços de saúde, bem como o fortalecimento da educação básica, para promover a saúde da referida população e prevenir o acometimento de parasitoses.

Palavras-chave: Educação em Saúde. Parasitos. Promoção da Saúde.

\section{ABSTRACT}

Introduction: Enteroparasites are diseases with a potential for cosmopolitan infection, which often affect age groups with weakened immunity, especially if the individuals are in an environment conducive to infection. However, this type of disease can be prevented through hygienic habits. Objective: To verify the knowledge about enteroparasites and the attitude of the children of Projeto Cidadão do Amanhã in the prevention of diseases. Methodology: This is a quasi-experimental study, carried out with children aged 6 to 12 years, enrolled in Projeto Cidadão do Amanhã and attended at the Nursing Clinic of the Federal University of Rio Grande do Norte, both in the city of Santa Cruz-RN . A sample of 40 children was used, two instruments being used for data collection. Results: The students' knowledge was verified in four categories: 1) Hygiene habits; 2) Food contamination; 3) Self-contamination and 4) Topical dermatitis, observing in the result of the post-test applied to group 2, improvement of correct responses, so that it is believed that health education is the main form of prevention of both enteroparasitosis and other diseases that may affect the life not only of the group studied here, but of the population in general. Conclusion: It is concluded that there is a need for implementation and implementation of health actions and services, as well as the strengthening of basic education, to promote the health of the population and prevent the involvement of parasitic diseases.

Keywords: Health Education; Parasites; Health Promotion. 


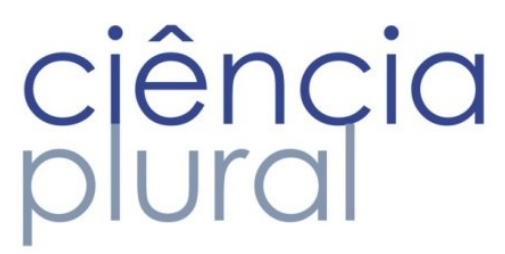

\section{INTRODUÇÃO}

As enteroparasitoses tratam-se de doenças acometidas por protozoários e/ou helmintos e representam um grave problema de saúde pública, particularmente nos países subdesenvolvidos, onde se apresentam bastante disseminadas e com alta prevalência, decorrente das más condições de vida das camadas populacionais mais carentes. As precárias condições socioeconômicas e higiênico-sanitárias são fatores importantes que contribuem para sua elevada prevalência no Brasil, haja vista que afetam grande parte dos indivíduos de todas as faixas etárias, especialmente as crianças ${ }^{1-4}$.

Segundo dados da Organização Mundial de Saúde (OMS), as doenças infecciosas e parasitárias continuam a figurar entre as principais causas de morte, uma vez que são responsáveis por 2 a 3 milhões de óbitos por ano, em todo o mundo. As parasitoses intestinais constituem um grave problema de saúde pública, principalmente na região Nordeste do Brasil que, apesar de alguns avanços nas últimas décadas, continua a apresentar elevados índices de mortalidade causados por doenças diarréicas, sobretudo entre indivíduos menores de cinco anos 5 .

A infecção por um ou vários parasitos intestinais é universal, por causa da disseminação desses agentes e da facilidade com que são transmitidos. A transmissão ocorre principalmente pela ingestão de água e alimentos contaminados com cistos e ovos de parasitos e pela penetração de larvas de helmintos na pele e nas mucosas. Na infância, a susceptibilidade às infecções parasitárias é mais elevada em razão de os hábitos de higiene ainda serem pouco consolidados. Além disso, a conglomeração humana nas escolas favorece a disseminação de agentes infecciosos ${ }^{6}$.

As crianças possuem uma imunidade relativamente baixa e um potencial consideravelmente alto de contaminação, e o meio onde ela estiver inserida estará totalmente ligado a sua saúde. Com isso, é possível dizer que a falta de saneamento básico, a condição socioeconômica da família desta criança, a educação sanitária que tenha recebido seu índice de massa corporal e suas funções metabólicas são fatores extremamente ligados ao seu sistema de defesa e, sobretudo, ao seu grau de contaminação. A desnutrição, por exemplo, é um forte fator para que aumente a possibilidade de infecções nesta faixa etária e é ainda um dos problemas causados pela contaminação por enteroparasitas.

As parasitoses intestinais prejudicam a absorção de nutrientes e são responsáveis pela baixa estatura da criança. A respeito dessas infecções, é importante ressaltar que, frequentemente, constituem situações muito simples, nas quais, em uma criança saudável não teriam grandes consequências, mas em crianças desnutridas poderiam prejudicar não só o ganho de peso como também a estatura². 


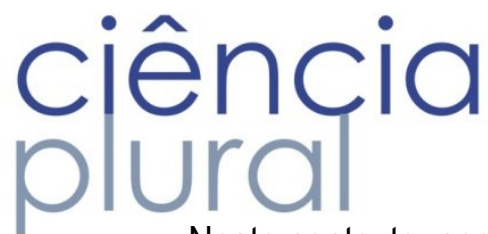

Neste contexto, por entender que a identificação de situações de vulnerabilidade como os casos de enteroparasitoses podem tornar os profissionais de saúde mais sensíveis com as demandas do cuidar e a garantia da efetividade de ações e serviços oferecidos, através do conhecimento das reais necessidades da população, este estudo objetivou descrever o conhecimento sobre enteroparasitas e as atitudes das crianças diante da prevenção das doenças dos participantes do Projeto Cidadão do Amanhã, na cidade de Santa Cruz/RN.

\section{METODOLOGIA}

Trata-se de uma pesquisa experimental, realizado com crianças de 6 a 12 anos cadastradas no Projeto Cidadão do Amanhã e atendidas na Clínica de Enfermagem da Universidade Federal do Rio Grande do Norte, ambas localizadas no bairro do Paraíso, cidade de Santa Cruz-RN, Brasil.

O presente estudo é um recorte da pesquisa intitulada: Conhecimento e atitudes sobre o cuidado com parasitoses intestinais em crianças, realizada no período de Junho à Novembro de 2013. Como critérios de inclusão, devia estar na faixa etária entre 6 e 12 anos de idade, ou seja, se enquadrar na fase pré-escolar e escolar; frequentar o Projeto cidadão do amanhã no Centro de Juventude João de Dula e ser atendido na Clínica de Enfermagem. Foi utilizada uma amostra piloto, com a representatividade de 40 crianças, escolhidas mediante critérios de inclusão, posteriormente divididas em dois grupos. Essa escolha amostral se deu na modalidade de piloto em virtude da ausência de informações sobre o conhecimento existente entre crianças para esse agravo.

Para coleta de dados foi utilizado dois instrumentos. O primeiro tratou-se de um formulário não validado, com figuras que representavam hábitos de vida e de higiene, elaborado pelas pesquisadoras e que esteve embasado nas observações realizadas na convivência das crianças do Projeto. 0 segundo instrumento foi usado apenas com o grupo 2, e trata-se de um formulário de intervenção educativa, também elaborado pelas pesquisadoras, e diz respeito a um jogo de tabuleiro não validado, confeccionado e montado em sala reservada no projeto social, de forma que as crianças puderam interagir sendo os piões do jogo, com hábitos corretos e errados de higiene.

Os dados foram tabelados e analisados no software Stata 9.0. Para análise dos dados foram calculadas as medidas de estatística descritiva como média, mediana e desvio-padrão, e foi realizada comparação entre os grupos 1 e 2. No âmbito da análise bivariada, foi aplicado teste qui-quadrado, convencionando-se como nível de significância uma probabilidade inferior à 0,05 e Intervalo de Confiança de 95\%, porém, não obteve-se correlação significativa.

O estudo seguiu todas as recomendações constantes da Resolução 466/2012 do Conselho Nacional de Saúde, referentes à pesquisa com seres humanos. O projeto foi submetido e aprovado pelo Comitê de 
Ética e Pesquisa (CEP), da Universidade Federal do Rio Grande do Norte (UFRN)/Faculdade de Ciências da Saúde do Trairi (FACISA), tendo como número de aprovação 348.897.

\section{RESULTADOS}

Para a realização deste estudo foram selecionados dois grupos, identificados por grupo 1 e grupo 2. Os dois grupos responderam ao pré-teste, no qual existiam 25 figuras, sendo 15 certas e 10 erradas, porém o grupo 2, além de responder ao pré-teste, participou de uma ação educativa e, em seguida, respondeu ao pós-teste. Vale salientar que a escolha do grupo que participaria da ação educativa foi aleatória.

Observou-se que a média de idade do grupo 1 foi de 10,25 anos; mediana de 11,00 e desvio padrão de 1,83. Já no grupo 2, a média foi de 9,85 anos; mediana de 10,00 e desvio padrão de 1,38. No que diz respeito ao gênero das crianças aqui estudadas, o grupo 1 constituía-se por $60 \%$ ( $n=12)$ do sexo feminino e 40\% (n=08) do sexo masculino. Já no grupo 2, foram $50 \%(n=10)$ do sexo feminino e $50 \%(n=10)$ do sexo masculino. Desse modo, a média de participação da amostra foi de escolares na pré-adolescência.

O presente estudo foi dividido em quatro categorias, que foram escolhidas durante a análise da interpretação das figuras, sendo: 1) Hábitos de higiene; 2) Contaminação alimentar; 3) Autocontaminação e 4) Dermatites tópicas.

Tabela 1 - Distribuição dos acertos referentes ao tópico hábitos de higiene, dos grupos 1 e 2.

Santa Cruz/RN, Brasil, 2014.

\begin{tabular}{|c|c|c|c|c|c|c|c|c|c|}
\hline \multirow[b]{2}{*}{ Variável } & \multicolumn{3}{|c|}{$\begin{array}{c}\text { Grupo } 01 \\
\text { (pré-teste) }\end{array}$} & \multicolumn{3}{|c|}{$\begin{array}{c}\text { Grupo } 02 \\
\text { (pré-teste) }\end{array}$} & \multicolumn{3}{|c|}{$\begin{array}{c}\text { Grupo } 02 \\
\text { (pós-teste) }\end{array}$} \\
\hline & Acertos & $n$ & $f$ & Acertos & $\mathrm{n}$ & $f$ & Acertos & $\mathrm{n}$ & $F$ \\
\hline Tomar banho & Sim & 15 & 75,0 & Sim & 19 & 95,0 & Sim & 20 & 100,0 \\
\hline Unhas limpas & Sim & 17 & 85,0 & Sim & 19 & 95,0 & Sim & 19 & 95,0 \\
\hline Cortar unhas das mãos & Sim & 17 & 85,0 & Sim & 18 & 90,0 & Sim & 20 & 100,0 \\
\hline Cortar unhas dos pés & Sim & 18 & 90,0 & Sim & 17 & 85,0 & Sim & 19 & 95,0 \\
\hline Lavar as mãos & Sim & 16 & 85,0 & Sim & 13 & 65,0 & Sim & 19 & 95,0 \\
\hline
\end{tabular}

Quanto ao grupo 1 (pré-teste), tabela 1, pode-se perceber que obtiveram os seguintes índices de acertos frente às figuras que representavam os hábitos saudáveis: tomar banho 15 (75,0\%), unhas limpas $17(85,0 \%)$, cortar as unhas das mãos $17(85,0 \%)$, cortar unhas dos pés $18(90,0 \%)$ e lavar as mãos 16 (80,0\%). Observou-se no grupo 2 (pré-teste), como acertos, os seguintes valores: tomar banho 19 (95,0\%), lavar os cabelos $18(90,0 \%)$, unhas limpas $19(95,0 \%)$, cortar as unhas das mãos $18(90,0 \%)$, cortar unhas dos pés $17(85,0 \%)$ e lavar as mãos $13(65,0 \%)$. 


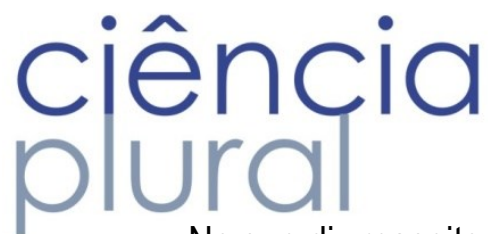

No que diz respeito ao grupo 2 no pós-teste, obteve-se o seguinte índice de acertos: tomar banho 20 (100,0\%), lavar os cabelos $19(95,0 \%)$, unhas limpas $19(95,0 \%)$, cortar as unhas das mãos $20(100,0 \%)$, corta unhas dos pés $19(95,0 \%)$ e lavar as mãos 19 (95,0\%).

Tabela 2 - Distribuição dos acertos referentes ao tópico contaminação alimentar, dos grupos $1 \mathrm{e}$ 2. Santa Cruz/RN, Brasil, 2014.

\begin{tabular}{ccccccccccc}
\hline & \multicolumn{2}{c}{$\begin{array}{c}\text { Grupo 01 } \\
\text { (pré-teste) }\end{array}$} & \multicolumn{3}{c}{$\begin{array}{c}\text { Grupo 02 } \\
\text { (pré-teste) }\end{array}$} & \multicolumn{3}{c}{$\begin{array}{c}\text { Grupo 02 } \\
\text { (pós-teste) }\end{array}$} \\
\hline Variável & Acertos & $\mathbf{n}$ & $\mathbf{f}$ & Acertos & $\mathbf{n}$ & $\mathbf{f}$ & Acertos & $\mathbf{n}$ & $\mathbf{f}$ \\
\hline Beber água da torneira & Não & 17 & 85,0 & Não & 18 & 90,0 & Não & 19 & 95,0 \\
\hline Lavar a maçã & Sim & 16 & 80,0 & Sim & 15 & 75,0 & Sim & 20 & 100,0 \\
\hline Lavar o tomate & $\operatorname{Sim}$ & 16 & 80,0 & Sim & 20 & 100,0 & Sim & 18 & 90,0 \\
\hline Lavar a alface & Sim & 19 & 95,0 & Sim & 19 & 95,0 & Sim & 19 & 95,0 \\
\hline
\end{tabular}

Quanto a contaminação alimentar, tabela 2, o grupo 1 (pré-teste) revela os seguintes resultados: beber água da torneira $17(85,0 \%)$, lavar a maçã $16(80,0 \%)$, lavar o tomate $16(80,0 \%)$ e lavar a alface 19 (95,0\%). No grupo 2 (pré-teste), observa-se que o nível de acerto foi nas variáveis: beber água da torneira $18(90,0 \%)$, lavar a maçã $15(75,0 \%)$, lavar o tomate $20(100,0 \%)$, enquanto lavar a alface $19(95,0 \%)$. Ainda, no que se refere aos acertos do grupo 2 (pós-teste), verificou-se: beber água da torneira 19 (95,0\%), lavar a maçã $20(100,0 \%)$, lavar o tomate $18(90,0 \%)$ e lavar a alface $19(95,0 \%)$.

Tabela 3 - Distribuição dos acertos referente à categoria auto contaminação dos grupos 1 e 2. Santa Cruz/RN, Brasil, 2014.

\begin{tabular}{cccccccccc}
\hline & \multicolumn{2}{c}{$\begin{array}{c}\text { Grupo 01 } \\
\text { (pré-teste) }\end{array}$} & \multicolumn{2}{c}{$\begin{array}{c}\text { Grupo 02 } \\
\text { (pré-teste) }\end{array}$} & \multicolumn{3}{c}{$\begin{array}{c}\text { Grupo 02 } \\
\text { (pós-teste) }\end{array}$} \\
\hline Variável & Acertos & $\mathbf{n}$ & $\mathbf{f}$ & Acertos & $\mathbf{n}$ & $\mathbf{f}$ & Acertos & $\mathbf{n}$ & $\mathbf{f}$ \\
\hline Roer unhas & Não & 19 & 95,0 & Não & 19 & 95,0 & Não & 19 & 95,0 \\
\hline Ter unhas sujas & Não & 17 & 80,0 & Não & 19 & 95,0 & Não & 19 & 95,0 \\
\hline Ter mãos sujas & Não & 20 & 100,0 & Não & 20 & 100,0 & Não & 20 & 100,0 \\
\hline Andar em esgoto & Não & 19 & 95,0 & Não & 19 & 95,0 & Não & 20 & 100,0 \\
\hline Andar sem calçados & Não & 20 & 100,0 & Não & 20 & 100,0 & Não & 19 & 95,0 \\
\hline Brincar na lama & Não & 18 & 90,0 & Não & 18 & 90,0 & Não & 20 & 100,0 \\
\hline
\end{tabular}

Referente à categoria auto contaminação, tabela 3 , quanto ao grupo 1 (pré-teste), aponta que obtevese os seguintes valores: roer unhas $19(95,0 \%)$, ter unhas sujas $17(80,0 \%)$, ter mãos sujas $20(100,0 \%)$, 


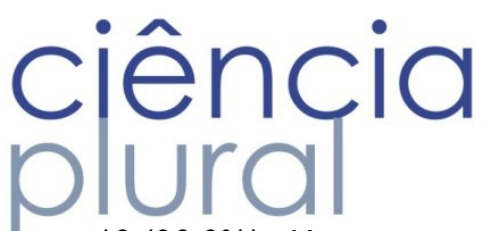

andar em esgoto 19 (95,0\%), andar sem calçados $20(100,0 \%)$ e brincar na lama com 18 (90,0\%). Já para o grupo 2 (pós-teste), quanto aos acertos, pode-se verificar os seguintes valores: roer unhas 19 (95,0\%), ter unhas sujas $19(95,0 \%)$, ter mãos sujas $20(100,0 \%)$, andar em esgoto $19(95,0 \%)$, andar sem calçados 20 $(100,0 \%)$ e brincar na lama $18(90,0 \%)$.

O pós-teste aplicado ao grupo 2 obteve os seguintes índices de acertos, de acordo com o descrito a seguir: roer unhas $95 \%(n=19)$, ter unhas sujas $95 \%(n=19)$, ter mãos sujas $100 \%(n=20)$, andar em esgoto $100 \%(n=20)$, andar sem calçado $95 \%(n=19)$ e brincar na lama 100\% $(n=20)$.

Tabela 4 - Distribuição dos acertos referentes à categoria dermatite tópica, dos grupos 1 e 2 . Santa Cruz/RN, Brasil, 2014.

\begin{tabular}{|c|c|c|c|c|c|c|c|c|c|}
\hline \multirow[b]{2}{*}{ Variável } & \multicolumn{3}{|c|}{$\begin{array}{l}\text { Grupo } 01 \\
\text { (pré-teste) }\end{array}$} & \multicolumn{3}{|c|}{$\begin{array}{c}\text { Grupo } 02 \\
\text { (pré-teste) }\end{array}$} & \multicolumn{3}{|c|}{$\begin{array}{c}\text { Grupo } 02 \\
\text { (pós-teste) }\end{array}$} \\
\hline & Acertos & $\mathrm{n}$ & $f$ & Acertos & $\mathrm{n}$ & $f$ & Acertos & $\mathbf{n}$ & $f$ \\
\hline Andar com sapato & Sim & 16 & 80,0 & Sim & 18 & 90,0 & Sim & 18 & 90,0 \\
\hline Brincar com sapato & Sim & 16 & 80,0 & Sim & 14 & 70,0 & Sim & 16 & 80,0 \\
\hline Roer unhas & Não & 19 & 95,0 & Não & 19 & 95,0 & Não & 19 & 95,0 \\
\hline Unhas sujas & Não & 16 & 80,0 & Não & 19 & 95,0 & Não & 19 & 95,0 \\
\hline Lavar as mãos & Sim & 16 & 80,0 & Sim & 13 & 65,0 & Sim & 19 & 95,0 \\
\hline Lavar o cabelo & Sim & 17 & 85,0 & Sim & 19 & 90,0 & Sim & 19 & 95,0 \\
\hline Usar chinelo & Não & 11 & 55,0 & Sim & 17 & 85,0 & Sim & 17 & 85,0 \\
\hline
\end{tabular}

Quanto a categoria dermatite tópica, tabela 4, o grupo 1 (pré-teste) obteve os seguintes índices de acertos: andar com sapato $16(80,0 \%)$, brincar com sapato $16(80,0 \%)$, roer unhas $19(95,0 \%)$, ter unhas sujas $16(80,0 \%)$, lavar as mãos $16(80,0 \%)$, lavar os cabelos $17(85,0 \%)$ e usar chinelo $11(55,0 \%)$. Para 0 grupo 2 (pré-teste) tem-se os seguintes resultados: andar com sapato $18(90,0 \%)$,brincar com sapato 14 (70,0\%), roer unhas $19(95,0 \%)$, ter unhas sujas 19 (95,0\%), lavar as mãos, $13(65,0 \%)$, lavar os cabelos $19(90,0 \%)$ e usar chinelo $17(85,0 \%)$. No pós-teste aplicado também ao grupo 2, obteve-se 0 resultado referente aos acertos: andar com sapato $18(90,0 \%)$, brincar com sapato $16(80,0 \%)$, roer unhas $19(95,0 \%)$, ter unhas sujas $19(95,0 \%)$, lavar as mãos $19(95,0 \%)$, lavar os cabelos $19(95,0 \%)$ e usar chinelos $17(85,0 \%)$.

\section{DISCUSSÃO}

Os resultados revelaram uma prevalência de crianças do sexo feminino (grupo 1), já o grupo 2 manteve uma paridade masculino e feminino. A idade das crianças escolares esteve próximo da faixa etária da adolescência. Buscou-se saber neste estudo os conhecimentos já adquiridos pelas crianças sobre os hábitos saudáveis de higiene e suas atitudes frente aos hábitos que visam prevenir as enteroparasitoses. 


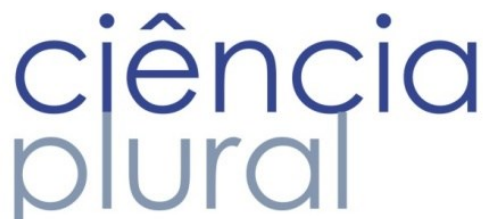

Ao realizar uma análise descritiva deste estudo, no resultado do pós-teste aplicado ao grupo 2, notou-se que houve melhoria de acertos das respostas. Os erros podem ter ocorrido em virtude da possibilidade da ocorrência do viés de memória, haja vista que muitas vezes a capacidade de apreensão do educando está atrelada ao grau de importância que ele dá ao fato ocorrido.

Considerando a categoria de higiene (tabela 01), vários estudos retratam que as formas erradas, falta dos hábitos de higiene pessoal e coletiva e, ainda, a falta de saneamento básico, o desconhecimento a respeito do mecanismo de infecção, que pode ter relação com o nível cultural dos envolvidos, são ditas como causa da contaminação por enteroparasitoses ${ }^{7-10}$.

Sabe-se que a deficiência na estrutura sanitária é um dos principais fatores para alta prevalência de enteroparasitoses. Assim, a contaminação por enteroparasitoses está diretamente relacionada com a falta de educação sanitária da população acometida. Então, pode haver a prevenção de tal contaminação através de mudanças nos hábitos de higiene, além da realização de ação pelos órgãos governamentais, que visem à melhoria da saúde dessa população.

No que diz respeito à contaminação alimentar (tabela 02), sabe-se que esta acontece por helmintos e protozoários, pela ingestão de hortaliças consumidas cruas, provenientes de áreas cultivadas e contaminadas por dejetos fecais. 12 Estudos retratam a importância da forma correta de manipular os alimentos e do devido tratamento que a água deve receber. Pois, segundo eles, a contaminação por enteroparasitas tem como principal fator a contaminação por via oral, através da ingestão de ovos ou cistos presentes em alimentos, água ou objetos contaminados por estas estruturas parasitarias ${ }^{7-10}$.

Portanto, fica claro que uma das formas que podem diminuir o índice de contaminação por enteroparasitoses é a reeducação na forma correta de manipular alimentos, atividade de extrema importância para prevenir não só a patologia em questão, como também seus agravos, uma vez que possibilita tanto para os manipuladores de alimentos, quanto para quem os consome, melhor qualidade de vida.

Ao referir-se à autocontaminação (tabela 03), a infecção por alguns geohelmintos como, por exemplo, Ascaris lumbricoides, também ocorre através do uso de água sem tratamento, contaminada com ovos, e não só através da ingestão de água contaminada, mas também através do banho, na higiene pessoal ou por alimentos contaminados. Porém, ocorre primordialmente por meio da contaminação do ambiente com ovos de helmintos no solo ou no piso das habitações. As precárias condições ambientais decorrentes da insalubridade das habitações são descritas como fatores favoráveis para o aumento das infecções por helmintos. 


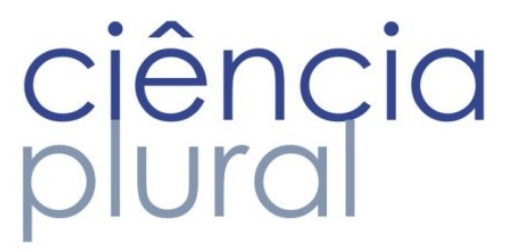

Ações como o contato direto e contínuo com o solo, a poeira, objetos ou partes do corpo que estejam contaminados e sejam levados à boca, além da falta do hábito de lavar as mãos antes das refeições ou após usar o banheiro, são fatores que contribuem diretamente na contaminação por enteroparasitoses ${ }^{7-10}$.

Dessa forma, pode-se notar que as ações supracitadas estão presentes no dia a dia da faixa etária aqui estudada, de maneira que a educação em saúde pode sim contribuir com a modificação desses hábitos. Nesse sentido, no que diz respeito a ações para crianças, fica claro que as atividades lúdicas contribuem para a facilidade de aquisição do conhecimento.

No que concerne à categoria de dermatite tópica (tabela 04), se sabe que a ocorrência de parasitoses vem causar dermatites devido à imunossupressão, causada pela falta de higiene, pela falha no sistema de saneamento básico, falhas na descontaminação e no abastecimento da água para consumo, e tem como principal sintoma o prurido, que é o principal causador das lesões cutâneas ${ }^{8}$. As irritações na pele são vistas como um dos principais sintomas determinantes causados pelas infecções parasitarias e que causam as dermatites acometidas, principalmente pela imunossupressão ocasionada pela contaminação parasitaria ${ }^{11}$.

Nessa perspectiva, nota-se que as parasitoses intestinais causam vários agravos à saúde da população afetada, sendo estes agravos causados especificamente pela imunossupressão advinda da patologia em questão. Por tanto, a fim de modificar não apenas a contaminação por enteroparasitoses, mas também as doenças oportunistas que modificam a qualidade de vida, do crescimento, do desenvolvimento e da cognição das crianças, mostram-se cada vez mais necessárias ações como a que aqui foi desenvolvida, visando à promoção e prevenção da saúde, em busca de uma melhor assistência e qualidade de vida para a população no geral.

$\mathrm{Na}$ atenção à saúde da criança, há estreita relação entre a Educação em Saúde e a promoção da saúde, visto que as ações implementadas em todos os níveis de atenção, além de tratar e/ou prevenir doenças, destinam-se também a promover o crescimento e o desenvolvimento infantil, numa perspectiva de qualidade de vida. As ações de promoção da saúde devem ser acionadas por meio de estratégias que envolvam a coletividade em geral e a família, que é a instituição responsável pela criança e detentora de um saber que não poderá ser descartado, mas aperfeiçoado e/ou adaptado ao saber científico dos profissionais $^{12}$.

As práticas de educação e saúde em uma proposta pautada pela construção compartilhada devem ser orientadas pela busca da interdisciplinaridade, da autonomia e da cidadania, de modo que privilegiem a interação comunicacional, onde sujeitos, detentores de saberes diferentes, apropriam-se destes, transformando-se e transformando-os ${ }^{13}$. 
Portanto, pode-se aqui dizer que a educação em saúde modifica os índices de morbidade das parasitoses. Entretanto, deve-se está pautado na interação profissional de saúde, educador e responsável, para que as crianças possam então colocar em prática todo o conhecimento adquirido em atividades que visam à modificação de hábitos da vida diária.

A ação educativa em saúde é um processo dinâmico que tem como objetivo a capacitação dos indivíduos e/ou grupos, em busca da melhoria das condições de saúde da população. Ressalta-se que, nesse processo, a população tem a opção de aceitar ou rejeitar as novas informações, podendo também adotar ou não novos comportamentos frente aos problemas de saúde. Não basta apenas seguir normas recomendadas de como ter mais saúde e evitar doenças, mas sim realizar a educação em saúde num processo que estimule o diálogo, a indagação, a reflexão, o questionamento e a ação partilhada ${ }^{14}$.

O estudo revela que os profissionais de saúde como, necessitam construir conhecimentos e planejar ações que orientem os educadores sobre quais cuidados devem ser realizados com a criança que frequenta instituições conhecidas como creche e, dessa forma, possa vir a prevenir agravos, dentre eles, as parasitoses ${ }^{15}$. Uma vez que sabe-se que em algumas instituições, as condições de aglomeração de crianças e de higiene do ambiente podem ser favoráveis à transmissão de alguns patógenos, dentre eles os enteroparasitos ${ }^{16}$.

Acredita-se que a educação em saúde seja a principal forma de prevenção tanto das enteroparasitoses, quanto de outras doenças que possam vir a afetar a vida não apenas do grupo aqui estudado, mas da população em geral. Pode-se, ainda, dizer que a pouca repetição de hábitos saudáveis de higiene pessoal e coletiva seja um dos fatores que contribuem diretamente com o elevado índice de morbidade da patologia aqui estudada. Todavia, por serem ações de fácil realização, que fazem parte do conhecimento de qualquer faixa etária, ainda é dada pouca importância para estas.

\section{CONCLUSÕES}

Avaliar o conhecimento de escolares quanto a atitudes que venham a prevenir as enteroparasitoses apresenta-se como fundamental para nortear as ações e serviços de saúde, com fins de reduzir os índices de acometimento de escolares por parasitoses. Quando foram explorados sobre seus conhecimentos na aplicação do pré- teste, notou-se que o conhecimento das crianças sobre as enteroparasitoses já existia, porém, a ausência de atitudes frente à prevenção das mesmas, que independe do conhecimento, mostrouse continuamente falha.

Quanto aos cuidados com as crianças, estas devem ser feitas de forma conjunta com ações que envolvam todos que fazem parte da educação infantil: pedagogos, familiares e os profissionais de saúde. Observa-se ainda a importância da realização de ações de intervenção realizadas com crianças usando 


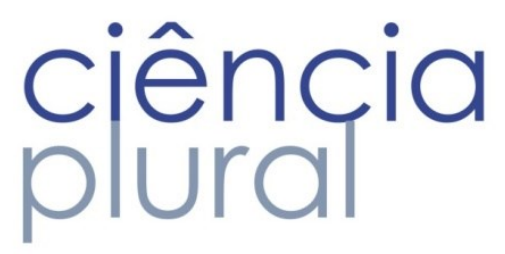

instrumentos lúdicos, para que venham a conquistar a atenção do público alvo de maneira prazerosa. Essas atividades devem estimular os participantes à reflexão, desenvolvendo um pensamento crítico sobre os temas trabalhados em seu dia a dia e as ações expostas durante a atividade realizada.

Ao serem explorados sobre seus conhecimentos na aplicação do pré-teste, notou-se que o conhecimento das crianças sobre as enteroparasitoses já estava presente, contudo a ausência de atitudes frente à prevenção das mesmas, que independe do conhecimento, mostrou-se continuamente falha, o que faz perceber que os conhecimentos existentes sobre a proteção causada pela higiene são ainda muito frágeis.

Portanto esse estudo revelou a necessidade de implantação e implementação de ações educativas, bem como o fortalecimento da educação básica, principalmente no que diz respeito às ações de saúde na escola, de modo que possa levar a mudanças dos hábitos de vida diários e multiplicar o conhecimento e as atitudes desta população para a promoção de saúde, através da continuidade de ações lúdicas, que visem à prevenção de morbidades, associando as ações erradas com as doenças que se podem adquirir. Desta maneira, são fortalecidos o processo de educação em saúde e as práticas de promoção de saúde na comunidade, o que redunda em melhorar as condições de saúde de forma geral.

\section{REFERÊNCIAS}

1. Frei $F$, Juncansen $C$, Ribeiro-Pae JT. Levantamento epidemiológico das parasitoses intestinais: viés analítico decorrente do tratamento profilático. Cad Saúde Pública. 2008; 24(12):2919-2925.

2. Silva EF, Silva EB, Almeida KS, Sousa JJN, Freitas FLC. Enteroparasitoses em crianças de áreas rurais do munícipio de Coari, Amazonas, Brasil. Revista de Patologia Tropical. 2009; 38(1):35-43.

3. Mati VLT, Pinto JH, Melo AL. Levantamento de parasitos intestinais nas áreas urbana e rural de Itambé do Mato Dentro, Minas Gerais, Brasil. Revista de Patologia Tropical. 2011; 40(1):92-100.

4. Borges WF, Marciano FM, Oliveira HB. Parasitos intestinais: elevada prevalência de giardialambliaem pacientes atendidos pelo serviço público de saúde da região sudeste de Goiás, Brasil. Revista de Patologia Tropical. 2011; 40(2):149-157.

5. Vasconcelos IAB, Oliveira JW, Cabral FRF, Coutinho HDM, Menezes IRA. Prevalência de parasitoses intestinais entre crianças de 4-12 anos no Crato, estado do Ceará: um problema recorrente de saúde pública. Acta Scientiarum Health Sciences. 2011; 33(1):35-41. 
6. Seixas MTL, Souza JN, Souza RP, Teixeira MCA, Soares NM. Avaliação da frequência de Parasitos Intestinais e do estado nutricional em escolares de uma área periurbana de salvador, Bahia, Brasil. Revista de Patologia Tropical. 2011; 40(4):304-314.

7. Andrade EC, Leite ICG, Rodrigues VO, Cesca MG. Parasitoses intestinais: uma revisão sobre seus aspectos sociais, epidemiológicos, clínicos e terapêuticos. Rev APS. 2010; 13(2):231-40.

8. Biasi LA, Tacca JA, Navarini M, Belusso R, Nardino A, Santolin JC, et al. Prevalência de enteroparasitoses em crianças de entidade assistencial de Erechim/RS. Perspectiva, Erechim. 2010; 34(125):173-9.

9. Oliveira VF, Amor ALM. Associação entre a ocorrência de parasitos intestinais e diferentes variáveis clínicas e epidemiológicas em moradores da comunidade Ribeira I, Araci, Bahia, Brasil. RBAC, 2012; 44(1):15-25.

10. Guilherme ALF, Araújo SM, Falavigna DLM, Pupulim ART, Dias MLGG, Oliveira HS, et al. Prevalência de enteroparasitas em horticultores e hortaliças da Feira do produtor de Maringá, Paraná. Revista da Sociedade Brasileira de Medicina Tropical, 1999; 32(4):405-11.

11. Joventino ES, Freitas LV, Rogério RF, Lima TM, Dias LMB, Ximenes LB. Jogo da memória como estratégia educativa para prevenção de enteroparasitoses: relato de experiência. Rev RENE. 2009; 10(2):141-148.

12. Queiroz MV, Jorge MS. Estratégias de Educação em Saúde e a qualidade do cuidar e ensinar em Pediatria: a interação, o vínculo e a confiança no discurso dos profissionais. Interface - Comunic, Saude, Educ. 2006; 10(19):117-30.

13. Acioli S, David HMSL, Faria MGA. Educação em saúde e a enfermagem em saúde coletiva: reflexões sobre a prática. Rev enferm UERJ, Rio de Janeiro, 2012 out/dez; 20(4):533-6.

14. Martins JJ, Albuquerque GL, Nascimento ERP, Barra DCC, Souza WGA, Pacheco WNS. Necessidades de educação em saúde dos cuidadores de pessoas idosas no domicílio. Texto contexto - enferm. 2007; 16(2): 254-62.

15. Castro CG, Beyrodt CGP. Ações de enfermagem na prevenção de parasitoses em creches. Rev Enferm UNISA. 2003; 4: 76-80.

16. Silva AVM, Corrêa BS, Carneiro IM, Pinto PR, Almeida AAP, Silva JH. Ações de extensão para promoção da saúde em creche no município de Belo Horizonte, MG. Interagir: pensando a extensão. 2016; $22: 32-45$. 